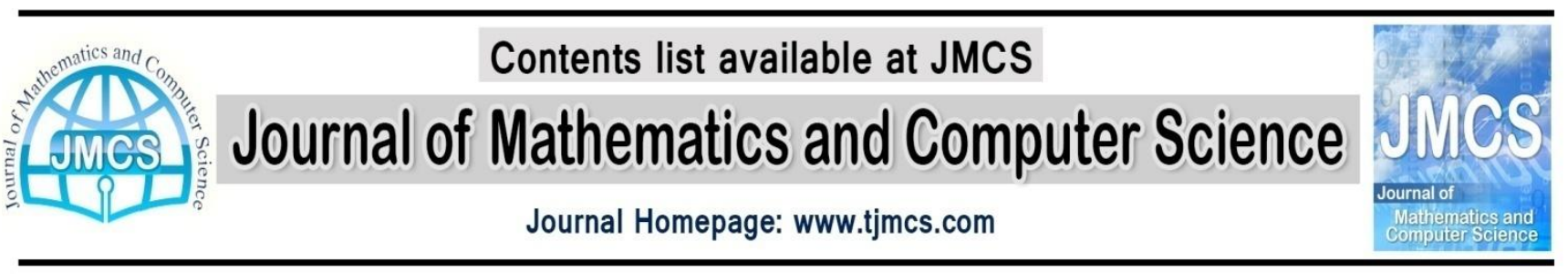

\title{
Compact Topological Semigroups associated with Oids
}

\author{
Abdol Mohammad Aminpour ${ }^{1}$, Mehrdad Seilani ${ }^{2}{ }^{2 *}$ \\ ${ }^{1}$ Department of Mathematical Sciences and Computer, Shahid Chamran University, Ahvaz, \\ Iran. \\ ${ }^{2}$ Iranian Academic Center for Education Culture and Research(ACECR) \\ "(Corresponding author)Tel: +98-9166024698,Email: Seilanimaths11@yahoo.com
}

\author{
Article history: \\ Received July 2014 \\ Accepted August 2014 \\ Available online August 2014
}

\begin{abstract}
The known theory for a discrete oid $T$ shows that how to find a subset $T^{\infty}$ of $\beta T$ which is a compact right topological semigroup (see section 2 for details).In this paper we try to find an analogue of almost periodic functions for oids. We discover, new compact semigroups by using a certain subspace of functions $\mathcal{U}^{\infty}(T)$ of $C(T)$ for an oid $T$ for which $f^{\beta}$ is continuous on $T^{\infty} \times\left(T \cup T^{\infty} \cup T T^{\infty}\right)$, where $\left(T \cup T^{\infty} \cup T T^{\infty}\right)$ is a suitable subspace of $\beta T$ for a wide range.

Mathematical Society Classification:2010, 54D35.

Keywords: Oid, Jointly continuous function ,Compact topological semigroup.
\end{abstract}

\section{Introduction}

Let $S$ be a semigroup and topological space. $S$ is called a topological semigroup if the multiplication $(s, t) \rightarrow s t: S \times S \rightarrow S$ is jointly continuous. Civin and Yood [4] shows that $\beta S$ the Stone-Čech compactification of a discrete semigroup $S$ could be given a semigroup structure, which need not be commutative on $S$ and is continuous in the left-hand variable; (that is for fixed $v \in \beta S$, the map $\mu \rightarrow \mu v: \beta S \rightarrow \beta S$ is continuous).Indeed the operation on $S$ extends uniquely to $\beta S$, so that $S$ contained in it's topological center [5]. Pym [7] introduced the concept of an oid (see Section 2 for precise definition). Oids are important because nearly all semigroups contain them and all oids are oid-isomorphic [6].We shall present our theory in a fairly concrete setting, so that our methods and results will be more readily accessible. Through out this paper we will let $T$ be a commutative standard oid with a discrete topology. Then the compact space $\beta T$ produces a compact right topological semigroup, "at 
infinity" $T^{\infty}$,so that its topological center is empty and it is not commutative(we refer the reader to [2],for these facts). Our aim of the present paper is to introduce a new compact topological semigroup for an oid $T$, using a certain space of functions on $T$ which have jointly continuous extensions on subspace $T^{\infty} \times\left(T \cup T^{\infty} \cup T T^{\infty}\right)$ of $T^{\infty} \times \beta T$ where $\left(T \cup T^{\infty} \cup T T^{\infty}\right)$ is a suitable subspace of $\beta T$ which is as large as possible. $C(T)$ is the $C^{*}$-algebra of all bounded continuous complex valued functions defined on the discrete space $T$ and $C(T)^{*}$ is the dual space of $C(T)$; we indicate the supremum norm on $C(T)$ by $\|$.$\| .We define a subset \mathcal{U}^{\infty}(T)$ containing all $f \in C(T)$ such that $f^{\beta}$ is jointly continuous on $T^{\infty} \times(T \cup$ $T^{\infty} \cup T T^{\infty}$ ) where $f^{\beta}$ is a unique continuous extension $f$ to $\beta T$. Then $\mathcal{U}^{\infty}(T)$ is a $C^{*}$-subalgebra of $C(T)$ (Lemma 3.3), so that $\mathcal{U}^{\infty}(T) \subseteq$ $W A P^{\infty}(T)$ (see[1],for definition). Indeed, $W A P^{\infty}(T)$ need not be a subset of $\mathcal{U}^{\infty}(T)$ (Example4.27). From the functions space $\mathcal{U}^{\infty}(T)$ we shall able to define an equivalence relation $\mathcal{R}_{U^{\infty}{ }_{(T)}}$ on $T^{\infty}$ by $\mu \mathcal{R}_{U^{\infty}(T)} v$ if and only if $f^{\beta}(\mu)=f^{\beta}(v)$ for all $f \in \mathcal{U}^{\infty}(T)$. This does determine a closed congruence relation on $T^{\infty}$ Which makes the quotient $T^{\infty} / \mathcal{R}_{\mathcal{U}^{\infty}(T)}$ a compact Hausdorff commutative topological semigroup which is a new semigroup to consider. Also, we conclude by establishing some properties of $T^{\infty} / \mathcal{R}_{U^{\infty}(T)}$, for example $\left(T^{\infty} / \mathcal{R}_{U^{\infty}(T)}\right)^{2}$ is not dense in $T^{\infty} / \mathcal{R}_{U^{\infty}(T)}\left(\right.$ Proposition 4.14), it contains $2^{c}$ idempotents (Theorem 5.4) and $K\left(T^{\infty} / \mathcal{R}_{\mathcal{U}^{\infty}(T)}\right)$, the minimal ideal of $T^{\infty} / \mathcal{R}_{U^{\infty}(T)}$ contains a free abelian group on $2^{c}$ generators (Theorem6.2).

\section{Definitions and preliminaries}

Let $x=(x(n))_{n \in \mathbb{N}}$ be any sequence consisting of $1^{\prime} s$ and $\infty^{\prime} s$. Write

$1.1=1,1 . \infty=\infty .1=1$. We define

$$
\operatorname{supp}(x(n))_{n \in \mathbb{N}}=\{n \in \mathbb{N}: x(n)=\infty\},
$$

and write

$$
T=\left\{(x(n))_{n \in \mathbb{N}}: \operatorname{supp}(x(n))_{n \in \mathbb{N}} \text { is finite and non - empty }\right\} .
$$

A commutative standard oid is the set $T$ together with the product $x y$ defined in $T$ if and only if(supp $x) \cap(\operatorname{supp} y)=\varnothing$ to be $(x(n) y(n))$. Thus the product $x(n) y(n)$ is required to be defined if and only if either $x(n) \operatorname{or} y(n)$ is 1. Obviously, the product in $T$ is associative where defined and $\operatorname{supp}(x y)=$ ( $\operatorname{supp} x) \cup(\operatorname{supp} y)$ whenever $x y$ is defined in $T$ (oids are discussed in [7]). Any commutative standard oid $T$ can be considered as $\bigoplus_{n=1}^{\infty}\{1, \infty\} \backslash$ $\{(1,1, \ldots, 1)\}$. We use epithet "standard"to indicate that the index set is $\mathbb{N}$ (in [7],oids could have any index set). For $x, y \in T$, supp $x<\operatorname{supp} y$ means that $n<m$ if $n \in \operatorname{supp} x$ and $m \in \operatorname{supp} y$, and supp $x_{\alpha} \rightarrow \infty$ for some net $\left(x_{\alpha}\right)$ in $T$ will mean that for arbitrary $k \in \mathbb{N}$ eventually $\min \left(\operatorname{supp} x_{\alpha}\right)>k$. Then for 
a fixed $t \in T$, eventually $\operatorname{supp} t<\operatorname{supp} x_{\alpha}$ and so eventually $t x_{\alpha}$ is defined in $T$. Write $u_{n}=(1,1, \ldots, \infty, 1,1, \ldots)$ (with $\infty$ in the $n$th place). Put $=\left\{u_{n}: n \in \mathbb{N}\right\}$. Then $U$ is countable subset of $T$. Moreover, any $x \in T$ can be written uniquely as a finite product $x=u_{i_{1}} u_{i_{2}} \ldots u_{i_{k}}$ with $i_{1}<i_{2}<\cdots<i_{k}$ supp $x=$ $\left\{i_{1}, \ldots, i_{k}\right\}$.The compact space $\beta T$ is the Stone-Čech compactification of the discrete space $T$ and if $f$ maps $T$ to some compact space, $f^{\beta}$ is the unique continuous extension of $f$ to $\beta T$. We define

$$
T^{\infty}=\left\{\mu \in \beta T: \mu=\lim _{\alpha} x_{\alpha} \text { with supp } x_{\alpha} \rightarrow \infty\right\} .
$$

Obviously, $T \cap T^{\infty}=\varnothing$. For $\mu \in \beta T, v \in T^{\infty}$ the product $\mu \nu \in C(T)^{*}$ is defined by $\mu \nu=\mu \circ L_{v}$, where $L_{v} f(t)=\lim _{\beta} f\left(t y_{\beta}\right)$, if $t \in T, f \in C(T)$ and $y_{\beta} \rightarrow v$ with supp $y_{\beta} \rightarrow \infty$. Then $L_{v} f \in C(T), L_{v} f(t)=\left(L_{t} f\right)^{\beta}(v)$. Further, $L_{v}$ is a bounded linear operator on $C(T)$. Of course $\mu \in \beta T$ is a bounded linear functional on $C(T)$, with $\|\mu\| \leq 1$, if $\mu(f)=f^{\beta}(\mu)$. In fact, the product $(\mu, v) \rightarrow \mu v: \beta T \times$ $T^{\infty} \rightarrow T^{\infty}$ is defined and is right continuous, and left continuity holds when $\mu=t \in T$ [1].Also $\mu \nu=\lim _{\alpha} \lim _{\beta} x_{\alpha} y_{\beta}$ where $\left(x_{\alpha}\right)$ is a net in $T$ with $x_{\alpha} \rightarrow \mu$. If $\mu \in T^{\infty}$, then $L_{\mu \nu}=L_{\mu} \circ L_{v}$, so that $(\mu, v) \rightarrow \mu v: T^{\infty} \times T^{\infty} \rightarrow T^{\infty}$ is a binary operation on $T^{\infty}$ relative to which that $T^{\infty}$ is a compact right topological semigroup. If $\subseteq T$, then $1_{A}$ denotes the indicator function of $A$, that is, the function whose value is 1 on $A$ and 0 on $T \backslash A$.

Remark2.1. For $v \in T^{\infty}$ and $\mu \in \beta T, v \mu$ can not always be defined in a standard oid $T$, if we require that multiplication is right continuous. This is true even if $\mu \in T$. If $z_{n}=u_{1} u_{2} \ldots u_{n}, n \in \mathbb{N}, u_{n} \in U$ and $z_{n_{i}} \rightarrow \lambda \in \beta T$ for some subnet $\left(z_{n_{i}}\right) \circ f\left(z_{n}\right)$, then for any $t \in T, \lim _{i} t z_{n_{i}}$ is not defined. But we can define $v \mu$ for standard oids only on a subset of $T^{\infty} \times \beta T$. This subset includes $T^{\infty} \times(T \cup$ $\left.T^{\infty}\right)$. Now let $x_{\alpha} \rightarrow \mu$ in $\beta T$ with $\operatorname{supp} x_{\alpha} \rightarrow \infty$ and let $\lambda=t \lambda^{\prime}$ where $t \in T$ ,$\lambda^{\prime} \in T^{\infty}$ such that $y_{\beta} \rightarrow \lambda^{\prime}$ with supp $y_{\beta} \rightarrow \infty$. Then eventually supp $t<$ supp $x_{\alpha}$ and for such $\alpha$,eventually supp $x_{\alpha}<\operatorname{supp} y_{\beta}$, so that eventually $t x_{\alpha} y_{\beta}$ is defined in $T$ and hence $\lim _{\alpha} \lim _{\beta}\left(t x_{\alpha} y_{\beta}\right)=t \mu \lambda^{\prime}\left(=\mu t \lambda^{\prime}\right)$ (see[1],Definition 3.5). Therefore, we can defined $\mu \lambda$ on $T^{\infty} \times\left(T \cup T^{\infty} \cup T T^{\infty}\right)$ , whenever $\left(T \cup T^{\infty} \cup T T^{\infty}\right)$ is a suitable subspace of $\beta T$ for a wide range.

Definition2.2.(i) The cardinal function is the map $c: T \rightarrow \mathbb{N}$ given by $c(x)=\operatorname{card}(\operatorname{supp} x)$ (that is, the number of elements of the support of $x$ ). Then $c$ extends to a unique continuous extension $c^{\beta}$ from $\beta T$ into the one-point compactification $\mathbb{N} \cup\{\infty\}$. If $(\operatorname{supp} x) \cap(\operatorname{supp} y)=\varnothing$ so that $x y$ is defined in,$(x y)=c(x)+c(y)$, and so for $\mu \in \beta T, v \in T^{\infty}$ then $c^{\beta}(\mu \nu)=$ $c^{\beta}(\mu)+c^{\beta}(v)$. Thus $c^{\beta}$ is a homomorphism on $T^{\infty}$. We denote $1 / c(x)$ by $h(x)$ for $x \in T$.

(ii) The length function is the map $l: T \rightarrow \mathbb{N}$ by letting $l(x)$ (The length Of support of $x$ ) be the integer $i_{k}-i_{1}+1$ where supp $x=\left\{i_{1}, \ldots, i_{k}\right\}$. 
Then $l$ extends to a unique continuous extension $l^{\beta}$ from $\beta T$ into the onepoint compactification $\mathbb{N} \cup\{\infty\}$. We denote $1 / l(x)$ by $r(x)$ for $x \in T$.

(iii) The $z$-function is the map $z: T \rightarrow \mathbb{Z}^{+}$by letting $z(x)$ be the largest number of consecutive $1^{\prime} s$ between min (supp $\left.x\right)$ and max (supp $\left.x\right)$. Then $z$ extends to a unique continuous extension $z^{\beta}$ from $\beta T$ into the one-point compactification $\mathbb{Z}^{+} \cup\{\infty\}$. We denote $1 / z(x)+1$ by $k(x)$ for $\in T$.

We next have some useful results which we will need later.

Proposition2.3.For $\mu \in T^{\infty}, v \in\left(T \cup T^{\infty} \cup T T^{\infty}\right)$ then $l^{\beta}(\mu v)=\infty$.

Proof. Let $v=t \in T$, and let $x_{\alpha} \rightarrow \mu$ for some net $\left(x_{\alpha}\right)$ in T with supp $x_{\alpha} \rightarrow \infty$. Then eventually supp $t<\operatorname{supp} x_{\alpha}$, so that eventually $l\left(t x_{\alpha}\right)=\infty$. Since $t x_{\alpha} \rightarrow t \mu$ in $\beta T$ and $l^{\beta}$ is continuous on $\beta T$, from which it follows that $l^{\beta}(\mu t)=l^{\beta}(t \mu)=\infty$. If $v \in T^{\infty}$, and $y_{\beta} \rightarrow v$ for some net $\left(y_{\beta}\right)^{\text {in }} T$ with supp $y_{\beta} \rightarrow \infty$, then $l^{\beta}(\mu v)=\lim _{\alpha} \lim _{\beta} l\left(x_{\alpha} y_{\beta}\right)=\infty$, by a similar reason. Suppose that $v=t \lambda, \lambda \in T^{\infty}$. Then $\mu \lambda \in T^{\infty}$, since $T^{\infty}$ is a semigroup, Hence $l^{\beta}(\mu v)=l^{\beta}(\mu t \lambda)=l^{\beta}(t \mu \lambda)=\infty$ and the result follows.

The next result is an immediate consequence of Definition2.2(ii), Proposition2.3.

Corollary2.4. For $\mu \in T^{\infty}, v \in\left(T \cup T^{\infty} \cup T T^{\infty}\right)$. Then $r^{\beta}(\mu v)=0$.

Proposition2.5.Let $\mu \in T^{\infty}, v \in\left(T \cup T^{\infty} \cup T T^{\infty}\right)$. Then $z^{\beta}(\mu v)=\infty$. Proof. This uses Definition 2.2(iii), the proof is parallel to that of Proposition 2.3.

Corollary2.6. Let $\mu \in T^{\infty}, v \in\left(T \cup T^{\infty} \cup T T^{\infty}\right)$. Then $k^{\beta}(\mu \nu)=0$. Proof is straightforward.

\section{Space of jointly continuous functions}

Our aim of the present section is to introduce a new kind of $C^{*}$-subalgebra of the $C^{*}$-algebra $C(T)$. In this section we try to find an analogue of almost periodic functions for oids.

Definition3.1. Let $T$ be a commutative standard oid. We use $\mathcal{U}^{\infty}(T)$ to denote the set of all bounded complex valued functions on $T$ for which $(\mu, v) \rightarrow f^{\beta}(\mu v): T^{\infty} \times\left(T \cup T^{\infty} \cup T T^{\infty}\right) \rightarrow \mathbb{C}$ is jointly continuous. Clearly $\mathcal{U}^{\infty}(T)$ is conjugate closed and contains all constant functions.

Example3.2.(i) Let $h=1 / c$ be as in Definition 2.2(i). Then by a routine 
argument we see that for $\mu \in T^{\infty}, v \in\left(T \cup T^{\infty} \cup T T^{\infty}\right), c^{\beta}(\mu \nu)=c^{\beta}(\mu)+$ $c^{\beta}(v)$, and so $(\mu, v) \rightarrow h^{\beta}(\mu v): T^{\infty} \times\left(T \cup T^{\infty} \cup T T^{\infty}\right) \rightarrow \mathbb{C} \quad$ is jointly continuous. Therefore $h \in \mathcal{U}^{\infty}(T)$.

(ii)Let $r=1 / l$ be as in Definition 2.2(ii). Then by Corollary 2.4, $r^{\beta}(\mu v)=0$ for $\mu \in T^{\infty}$ and $v \in\left(T \cup T^{\infty} \cup T T^{\infty}\right)$, and so $(\mu, v) \rightarrow r^{\beta}(\mu v): T^{\infty} \times\left(T \cup T^{\infty} \cup\right.$ $\left.T T^{\infty}\right) \rightarrow \mathbb{C}$ is jointly continuous. Thus $r \in \mathcal{U}^{\infty}(T)$.

(iii) Let $k=1 / z+1$ be as in Definition 2.2(iii).Then by Corollary 2.6, $k^{\beta}(\mu v)=0$ for $\mu \in T^{\infty}, \quad v \in\left(T \cup T^{\infty} \cup T T^{\infty}\right)$, and $s o(\mu, v) \rightarrow k^{\beta}(\mu v): T^{\infty} \times\left(T \cup T^{\infty} \cup\right.$ $\left.T T^{\infty}\right) \rightarrow \mathbb{C}$ is jointly continuous, hence $k \in \mathcal{U}^{\infty}(T)$.

Lemma3.3. $U^{\infty}(T)$ is a $C^{*}$-subalgebra of the $C^{*}$-algebra $C(T)$.

Proof. It is easily seen that $\mathcal{U}^{\infty}(T)$ is a subalgebra of the algebra $C(T)$. To prove that $\mathcal{U}^{\infty}(T)$ is a $C^{*}$-subalgebra it is enough to prove that $\mathcal{U}^{\infty}(T)$ is a closed subalgebra of $C(T)$ because the other conditions are satisfied easily. For this purpose, let $\left(f_{n}\right)_{n \in \mathbb{N}}$ be any sequence in $\mathcal{U}^{\infty}(T), f \in C(T)$ with $\left\|f_{n}-f\right\| \rightarrow 0$, as $n \rightarrow \infty$. Suppose that $\mu_{\alpha} \rightarrow \mu$ in $T^{\infty}, v_{\alpha} \rightarrow v$ in $\left(T \cup T^{\infty} \cup\right.$ $T T^{\infty}$ ). Then given $\varepsilon>0$, choose $k \in \mathbb{N}$ such that $\left\|f_{n}-f\right\|<\varepsilon / 3$ for all $n \geq k$. Fix $n_{0}>k$. Then choose $\alpha_{0}$ such that $\alpha>\alpha_{0}$,

$$
\begin{aligned}
& \left|f_{n_{0}}^{\beta}\left(\mu_{\alpha} v_{\alpha}\right)-f_{n_{0}}^{\beta}(\mu \nu)\right|<\varepsilon / 3 . \text { For such } \alpha \text {, then } \\
& \begin{array}{c}
\left|f^{\beta}\left(\mu_{\alpha} v_{\alpha}\right)-f^{\beta}(\mu \nu)\right| \leq\left|f^{\beta}\left(\mu_{\alpha} v_{\alpha}\right)-f_{n_{0}}^{\beta}\left(\mu_{\alpha} v_{\alpha}\right)\right|+\left|f_{n_{0}}^{\beta}\left(\mu_{\alpha} v_{\alpha}\right)-f_{n_{0}}^{\beta}(\mu \nu)\right| \\
\quad+\left|f_{n_{0}}^{\beta}(\mu \nu)-f^{\beta}(\mu \nu)\right| \\
\leq\left\|f^{\beta}-f_{n_{0}}^{\beta}\right\|+\left|f_{n_{0}}^{\beta}\left(\mu_{\alpha} v_{\alpha}\right)-f_{n_{0}}^{\beta}(\mu \nu)\right|+\left\|f_{n_{0}}^{\beta}-f^{\beta}\right\| \\
<\varepsilon / 3+\varepsilon / 3+\varepsilon / 3=\varepsilon .
\end{array}
\end{aligned}
$$

Hence $\lim _{\alpha} f^{\beta}\left(\mu_{\alpha} v_{\alpha}\right)=f^{\beta}(\mu v)$ and so $(\mu, v) \rightarrow f^{\beta}(\mu v): T^{\infty} \times\left(T \cup T^{\infty} \cup\right.$ $\left.T T^{\infty}\right)$ is jointly continuous, as desired.

Our next result will be useful in later.

Theorem3.4. Let $f \in \mathcal{U}^{\infty}(T), \eta \in T^{\infty}$. Then $L_{\eta} f \in \mathcal{U}^{\infty}(T)$.

Proof. It is easy to check that $v \eta \in\left(T \cup T^{\infty} \cup T T^{\infty}\right)$ whenever $v \in\left(T \cup T^{\infty} \cup\right.$ $\left.T T^{\infty}\right)$. From this and that the product $(\mu, v) \rightarrow \mu v: \beta T \times T^{\infty} \rightarrow \beta T$ is right continuous, it follows that the map $v \rightarrow v \eta$ is continuous of $\left(T \cup T^{\infty} \cup T T^{\infty}\right)$ into itself. Therefore the composite map $(\mu, v) \rightarrow(\mu, v \eta) \rightarrow f^{\beta}(\mu \nu \eta)$ is continuous from $T^{\infty} \times\left(T \cup T^{\infty} \cup T T^{\infty}\right)$ to $\mathbb{C}$ for each $f \in \mathcal{U}^{\infty}(T)$. Thus

$$
f^{\beta}(\mu \nu \eta)=\mu \nu \eta(f)=\mu \nu \circ L_{\eta}(f)=\mu \nu\left(L_{\eta} f\right)=\left(L_{\eta} f\right)^{\beta}(\mu \nu) .
$$

It follows that, $(\mu, v) \rightarrow\left(L_{\eta} f\right)^{\beta}(\mu v): T^{\infty} \times\left(T \cup T^{\infty} \cup T T^{\infty}\right) \rightarrow \mathbb{C}$ is continuous, and therefore by Definition $3.1, L_{\eta} f \in \mathcal{U}^{\infty}(T)$, as desired.

Definition3.5. For $f \in \mathcal{U}^{\infty}(T)$ and $\quad v \in\left(T \cup T^{\infty} \cup T T^{\infty}\right)$, we define $L_{v} f^{\beta}(\mu)=f^{\beta}(\mu v)$, where $\mu \in T^{\infty}$.

Remark3.6.It is easy to check that $L_{v} f^{\beta}$ is continuous on compact space 
$T^{\infty}$, since $(\mu, v) \rightarrow \mu v: \beta T \times T^{\infty} \rightarrow \beta T$ is right continuous and left continuity holds when $\mu=t \in T$.Moreover, $L_{v}$ is a bounded linear operator with $\left\|L_{v}\right\| \leq 1$.

Theorem3.7.Let $f \in C(T)$. Then $(\mu, v) \rightarrow f^{\beta}(\mu v): T^{\infty} \times\left(T \cup T^{\infty} \cup T T^{\infty}\right) \rightarrow \mathbb{C}$ is jointly continuous if and only if $v \rightarrow L_{v} f^{\beta}:\left(T \cup T^{\infty} \cup T T^{\infty}\right) \rightarrow C\left(T^{\infty}\right)$ is norm continuous.

Proof. Define $\psi: T^{\infty} \times\left(T \cup T^{\infty} \cup T T^{\infty}\right) \rightarrow \mathbb{C}$ by $\psi(\mu, v)=f^{\beta}(\mu v)$. Then $\psi$ Is a bounded function, since $f^{\beta}$ is continuous on $\beta T$. It follows readily that $\mu \rightarrow \psi(\mu, v): T^{\infty} \rightarrow \mathbb{C}$ is a continuous function for each $v \in\left(T \cup T^{\infty} \cup T T^{\infty}\right)$. Let $C\left(T^{\infty}\right)$ have the uniform norm. Since $T^{\infty}$ is a compact space and $\left(T \cup T^{\infty} \cup T T^{\infty}\right)$ is a subspace of $\beta T, \psi$ is jointly continuous if and only if the mapping $\quad v \rightarrow \psi(0, v):\left(T \cup T^{\infty} \cup T T^{\infty}\right) \rightarrow C\left(T^{\infty}\right)$ is continuous (see[10] , Chapter 1, Lemma 1.8(a)).

Lemma3.8.If $v \rightarrow L_{v} f^{\beta}: T^{\infty} \rightarrow C\left(T^{\infty}\right)$ is norm-continuous, then $\left\{L_{v} f^{\beta}: v \in T^{\infty}\right\}$ is relatively norm compact in $C\left(T^{\infty}\right)$

Proof is straightforward.

The next result is an immediate consequence of Definition 3.1, Theorem 3.7 and Lemma3.8.

Corollary3.9. Let $f \in \mathcal{U}^{\infty}(T)$. Then $\left\{L_{v} f^{\beta}: v \in T^{\infty}\right\}$ is a norm relatively compact in $C\left(T^{\infty}\right)$.

\section{Compact topological semigroups}

In this section by starting with $\mathcal{U}^{\infty}(T)$ we will produce a new compact Commutative topological semigroup, and make an investigation of it's properties.

Assume that $\tau$ is the topology induced on a compact right topological Semigroup $T^{\infty}$ by $\beta T$ and $\tau_{\mathcal{U}^{\infty}(T)}$ is the weak topology induced on $T^{\infty}$ by the family $\left\{f^{\beta}: f \in \mathcal{U}^{\infty}(T)\right\}$. Then the identity map from $\left(T^{\infty}, \tau\right)$ onto $\left(T^{\infty}, \tau_{\mathcal{U}^{\infty}(T)}\right)$ is continuous, thus $\left(T^{\infty}, \tau_{U^{\infty}(T)}\right)$ is compact [9].

Definition4.1. For $\mu, v \in T^{\infty}$, define $\mu \mathcal{R}_{\mathcal{U}^{\infty}(T)} v$ if and only if $f^{\beta}(\mu)=f^{\beta}(v)$ For all $f \in \mathcal{U}^{\infty}(T)$. Clearly $\mathcal{R}_{\mathcal{U}^{\infty}(T)}$ is a closed relation on $\left(T^{\infty}, \tau_{U^{\infty}(T)}\right)$.

Remark4.2.It should be noted from Definition 3.1,It follows that if $f \in \mathcal{U}^{\infty}(T),(\mu, v) \rightarrow f^{\beta}(\mu v): T^{\infty} \times\left(T \cup T^{\infty}\right) \rightarrow \mathbb{C}$ is separately continuous, so that $f^{\beta}(\mu \nu)=f^{\beta}(v \mu)$ for all $\mu, v \in T^{\infty}$. Therefore $f \in W_{1}^{\infty}(T)$ (see [1], for details). 
Proposition4.3. $\mathcal{R}_{\mathcal{U}^{\infty}(T)}$ Is a congruence relation on $T^{\infty}$.

Proof. To prove that $\mathcal{R}_{\mathcal{U}^{\infty}(T)}$ is congruence, we use Remark 4.2 and Theorem 3.4.

Let $\mu \mathcal{R}_{U^{\infty}(T)} \mu^{\text {and }} v \mathcal{R}_{U^{\infty}(T)} v^{\prime \text { where }} \mu, v, \mu^{\prime}, v^{\prime} \in T^{\infty}$.

Pick $f \in \mathcal{U}^{\infty}(T)$. Then

$$
\begin{gathered}
f^{\beta}(\mu v)=\left(L_{v} f\right)^{\beta}(\mu)=\left(L_{v} f\right)^{\beta}\left(\mu^{\prime}\right)=f^{\beta}\left(\mu^{\prime} v\right)=f^{\beta}\left(v \mu^{\prime}\right)=\left(L_{\mu^{\prime}} f\right)^{\beta}(v) \\
=\left(L_{\mu^{\prime}} f\right)^{\beta}\left(v^{\prime}\right)=f^{\beta}\left(v^{\prime} \mu^{\prime}\right)=f^{\beta}\left(\mu^{\prime} v^{\prime}\right)
\end{gathered}
$$

Thus $\mu \nu \mathcal{R}_{U^{\infty}(T)} \mu^{\prime} v^{\prime}$, as claimed.

Proposition4.4. $\left(T^{\infty}, \tau_{\mathcal{U}^{\infty}(T)}\right)$ Is a compact topological semigroup.

Proof. We know that $\left(T^{\infty}, \tau_{U^{\infty}(T)}\right)$ is a compact space. Now let $\left(\mu_{\alpha}\right)$ be a net in $T^{\infty}$ converging to $\mu \operatorname{in}\left(T^{\infty}, \tau_{u^{\infty}(T)}\right)$. Then $\mu_{\alpha_{\beta}} \rightarrow \mu_{0}$ in $\left(T^{\infty}, \tau\right)$ for some subnet $\left(\mu_{\alpha_{\beta}}\right)$ of $\left(\mu_{\alpha}\right)$. Since identity map from $\left(T^{\infty}, \tau\right)$ onto $\left(T^{\infty}, \tau_{\mathcal{U}^{\infty}(T)}\right)$ is continuous, it follows that $\mu_{\alpha_{\beta}} \rightarrow \mu_{0}$ in $\left(T^{\infty}, \tau_{\mathcal{U}^{\infty}(T)}\right)$. Hence for each $f \in \mathcal{U}^{\infty}(T), f^{\beta}\left(\mu_{0}\right)=\lim _{\beta} f^{\beta}\left(\mu_{\alpha_{\beta}}\right)=f^{\beta}(\mu)$. So $\mu_{0} \mathcal{R}_{U^{\infty}(T)} \mu$. Similarly if $v_{\alpha} \rightarrow v$ and $v_{\alpha_{\beta}} \rightarrow v_{0}$ in $\left(T^{\infty}, \tau_{u^{\infty}(T)}\right)$ then $v_{0} \mathcal{R}_{U^{\infty}(T)} v$. Hence, as $\mathcal{R}_{U^{\infty}(T)}$ is a congruence relation on $T^{\infty}$ by Proposition 4.3, then $\mu_{0} v_{0} \mathcal{R}_{\mathcal{U}^{\infty}(T)} \mu v$, so that $f^{\beta}\left(\mu_{0} v_{0}\right)=f^{\beta}(\mu \nu)$ for all $f \in \mathcal{U}^{\infty}(T)$. Now let $\mu_{\alpha} \rightarrow \mu, v_{\alpha} \rightarrow \operatorname{vin}\left(T^{\infty}, \tau_{\mathcal{U}^{\infty}(T)}\right)$, let $\left(\mu_{\alpha_{\beta}} v_{\alpha_{\beta}}\right)$ be a subnet of $\left(\mu_{\alpha} v_{\alpha}\right)$. Using compactness of $\left(T^{\infty}, \tau\right)$ we find $\operatorname{subnets}\left(\mu_{\alpha_{\beta_{\gamma}}}\right),\left(v_{\alpha_{\beta_{\gamma}}}\right)$ with $\mu_{\alpha_{\beta_{\gamma}}} \rightarrow \mu_{0}, v_{\alpha_{\beta_{\gamma}}} \rightarrow v_{0}$ in $\left(T^{\infty}, \tau\right)$. Then for each $f \in \mathcal{U}^{\infty}(T)$, we have $\lim _{\gamma} f^{\beta}\left(\mu_{\alpha_{\beta_{\gamma}}} v_{\alpha_{\beta_{\gamma}}}\right)=f^{\beta}\left(\mu_{0} v_{0}\right)=f^{\beta}(\mu \nu)$, since $(\mu, v) \rightarrow f^{\beta}(\mu \nu): T^{\infty} \times\left(T \cup T^{\infty} \cup T T^{\infty}\right) \rightarrow \mathbb{C}$ is jointly continuous, hence $\mu_{\alpha} v_{\alpha} \rightarrow \mu v$, as required.

Corollary4.5. Let the quotient semigroup $T^{\infty} / \mathcal{R}_{U^{\infty}(T)}$ have the quotient topology. Then $T^{\infty} / \mathcal{R}_{\mathcal{U}^{\infty}(T)}$ is compact Hausdorff topological semigroup.

Proof. Use Definition 4.1 and Proposition 4.3.

Corollary4.6. $T^{\infty} / \mathcal{R}_{U^{\infty}(T)}$ is a commutative semigroup.

Proof. Take $\mu, v \in T^{\infty}$ and $f \in \mathcal{U}^{\infty}(T)$. Then $f^{\beta}(\mu \nu)=f^{\beta}(v \mu)$ (Remark 4.2), and thus $\mu \nu \mathcal{R}_{U^{\infty}(T)} v \mu$, which implies the assertion. on $T^{\infty} / \mathcal{R}_{\mathcal{U}^{\infty}(T)}$.

We conclude this section with some results (both algebraic, topological)

Theorem 4.7.K( $\left(T^{\infty} / \mathcal{R}_{U^{\infty}(T)}\right)$ the minimal ideal of $T^{\infty} / \mathcal{R}_{U^{\infty}(T)}$ Is compact topological group. 
Proof. This follows from Corollaries 4.5,4.6 and Corollary 1.5.3[3].

Remark4.8. For each $n \in \mathbb{N}$, write $H_{n}=\left\{\mu \in T^{\infty}: c^{\beta}(\mu)=n\right\}$ and $H_{\infty}=\{\mu \in$ $T^{\infty}: c^{\beta}(\mu)=\infty$ \}.Then $T^{\infty}=H_{1} \cup H_{2} \cup \ldots \cup H_{n} \cup \ldots \cup H_{\infty}$.

Hence $H_{n}$ is clopen and each $\mu \in H_{n}$ is a limit of a net $\left(x_{\alpha}\right)$ in $T$ withc $\left(x_{\alpha}\right)=$ nfor each $\alpha$. Further, $H_{n} H_{m} \subseteq H_{n+m}$ for all $m, n \in \mathbb{N}$, so $H_{1} \cup H_{2} \cup \ldots \cup H_{n} \cup \ldots$ is a sub semigroup of $T^{\infty}$. Recall that by Definition $2.2(i i), l$ is the length function and $r(x)=1 / l(x), x \in T$.

Lemma4.9.Let $\xi \in H_{n}$ for some $n \in \mathbb{N}$ with $l^{\beta}(\xi)<\infty$, let $\pi: T^{\infty} \rightarrow T^{\infty} / \mathcal{R}_{U^{\infty}(T)}$ be the quotient map. Then $\pi(\xi)$ is not a product. Proof. Let $g_{l} \pi=l^{\beta}$. Then $g_{l}$ is a continuous functions on $T^{\infty} / \mathcal{R}_{U^{\infty}(T)}$, since $r=1 / l$ and $r \in \mathcal{U}^{\infty}(T)$ (Example 3.2 (ii)) and that $T^{\infty} / \mathcal{R}_{\mathcal{U}^{\infty}(T)}$ have the quotient topology (see [9], Chapter 3, Theorem 9). If $\pi(\xi)=\pi\left(\xi_{1}\right) \pi\left(\xi_{2}\right)$ for some $\xi_{1}, \xi_{2} \in T^{\infty}$, then $l^{\beta}(\xi)=l^{\beta}\left(\xi_{1} \xi_{2}\right)=\infty$ (Proposition2.3), which contradicts $l^{\beta}(\xi)<\infty$.

Theorem4.10. $T^{\infty} / \mathcal{R}_{U^{\infty}(T)}$ has no identity. Proof. If $\pi(e)$ is an identity element for $T^{\infty} / \mathcal{R}_{U^{\infty}(T)}$, where $e \in T^{\infty}$, then $\pi(\xi)=\pi(e) \pi(\xi)=\pi(\xi) \pi(e)$ for all $\xi \in T^{\infty}$, which is impossible by Lemma 4.9.

Remark 4.11. It is easy to verify that, $l^{\beta}(e)=\infty$, whenever $\pi(e)$ is an idempotent in $T^{\infty} / \mathcal{R}_{\mathcal{U}^{\infty}(T)}$. We denote the set of all idempotents in $T^{\infty} / \mathcal{R}_{\mathcal{U}^{\infty}(T)}$ by $E\left(T^{\infty} / \mathcal{R}_{U^{\infty}(T)}\right)$. Thus we obtain that $E\left(T^{\infty} / \mathcal{R}_{U^{\infty}(T)}\right) \subseteq\left\{\pi(\xi): \xi \in T^{\infty}, l^{\beta}(\xi)=\right.$ $\infty\}$.

Proposition4.12. Let $\xi \in H_{n}$ for some $n \in \mathbb{N}$ withl ${ }^{\beta}(\xi)<\infty$. Then $\pi(\xi)$ Is not a left zero.

Proof. Left zeros are idempotents and we saw above that $l^{\beta}(\xi)=\infty$ if $\xi$ is an idempotent.

We next have the following theorem.

Theorem4.13. $T^{\infty} / \mathcal{R}_{U^{\infty}(T)}$ is not a left zero semigroup.

Proposition4.14. $\left(T^{\infty} / \mathcal{R}_{U^{\infty}(T)}\right)^{2}$ is not dense in $T^{\infty} / \mathcal{R}_{U^{\infty}(T)}$. Proof. Let $g_{r} \pi=r^{\beta}$, where $\pi: T^{\infty} \rightarrow T^{\infty} / \mathcal{R}_{U^{\infty}(T)}$ is the quotient map. Then $g_{r}$ is continuous on $T^{\infty} / \mathcal{R}_{\mathcal{U}^{\infty}(T)}$, since $r \in \mathcal{U}^{\infty}(T)$ (Example 3.2 (ii)), and that $T^{\infty} / \mathcal{R}_{U^{\infty}(T)}$ have the quotient topology. By Corollary 2.4,

$$
\left(T^{\infty} / \mathcal{R}_{U^{\infty}(T)}\right)^{2} \cap g_{r}^{-1}(0,1)=\varnothing
$$

But $g_{r}^{-1}(0,1)$ is a non-empty open set in $T^{\infty} / \mathcal{R}_{\mathcal{U}^{\infty}(T)}$, as claimed. 
Remark4.15. Let $\mu_{0} \in T^{\infty}$ be the cluster point of the sequence $\left(u_{n} u_{n+3}\right)_{n=1}^{\infty}$ in $\beta T$, where $u_{n} \in U$ for all $n \in \mathbb{N}$,let $g_{r} \pi=r^{\beta}$ (Proposition 4.14). Then $r^{\beta}\left(\mu_{0}\right)=1 / 4$ and since by Corollary 2.4, $r^{\beta}\left(\left(T^{\infty}\right)^{2}\right)=0$ which implies that $\pi\left(\mu_{0}\right)$ is not the limit of a net of elements $\left(T^{\infty} / \mathcal{R}_{\mathcal{U}^{\infty}(T)}\right)^{2}$. Thus we obtain an alternative proof of the Proposition 4.14 which will be required in the next result.

Theorem4.16. $T^{\infty} / \mathcal{R}_{U^{\infty}(T)}$ is not a left (resp, right) simple semigroup.

Proof. Indeed, $T^{\infty} / \mathcal{R}_{U^{\infty}(T)} \pi\left(\mu_{0}\right) \subseteq\left(T^{\infty} / \mathcal{R}_{U^{\infty}(T)}\right)^{2}$, and $T^{\infty} / \mathcal{R}_{U^{\infty}(T)} \pi\left(\mu_{0}\right)$ is closed in $T^{\infty} / \mathcal{R}_{U^{\infty}(T)}$. Thus $T^{\infty} / \mathcal{R}_{U^{\infty}(T)} \pi\left(\mu_{0}\right) \neq T^{\infty} / \mathcal{R}_{U^{\infty}(T)}$ by Proposition 4.14, so $T^{\infty} / \mathcal{R}_{U^{\infty}(T)}$ is not a left simple semigroup. Thus $T^{\infty} / \mathcal{R}_{\mathcal{U}^{\infty}(T)}$ is not a group (see [3], for more details).

From Theorem 4.16 and Definition 1.5.6[3], we get the following result.

Corollary4.17. $T^{\infty} / \mathcal{R}_{U^{\infty}(T)}$ is not topologically left (resp, right) simple.

Corollary4.18. $T^{\infty} / \mathcal{R}_{U^{\infty}(T)}$ is not cancellative (and hence is not group). Proof. Use Theorem 4.16 and Corollaries 3.13,3.14[3].

Remark4.19.If for each $k \in \mathbb{N}$, let $x_{m}^{(k)}=u_{m} u_{m+1} \ldots u_{m+k-1}, m \in \mathbb{N}$ and $y_{n}=u_{n} u_{n+1} \ldots u_{n^{2}} . n \in \mathbb{N}$, where $u_{n} \in U$ for all $n$, then $\operatorname{supp} x_{m}^{(k)} \rightarrow \infty$, supp $y_{n} \rightarrow \infty$. Let $\mu^{(k)}, v \in T^{\infty}$ be the cluster points of $\left(x_{m}^{(k)}\right)_{m=1}^{\infty},\left(y_{n}\right)_{n=1}^{\infty}$ In $\beta T$ respectively. Then $l^{\beta}\left(\mu^{(k)}\right)=k, l^{\beta}(v)=\infty$. Now, suppose that $g_{l} \pi=l^{\beta}$ (Lemma 4.9). Then $g_{l} \pi\left(\mu^{(k)}\right)=l^{\beta}\left(\mu^{(k)}\right)=k, g_{l} \pi(v)=l^{\beta}(v)=\infty$, which implies that $g_{l}$ and $l^{\beta}$ map $T^{\infty} / \mathcal{R}_{U^{\infty}(T) \text { and }} T^{\infty}$ onto the one-point compactification $\mathbb{N} \cup\{\infty\}$ respectively.

Next we shall prove the following result.

Proposition 4.20. $\left(g_{l}^{-1}(\infty)\right)^{2}$ is not dense in $g_{l}^{-1}(\infty)$ (and hence isnot dense in $T^{\infty} / \mathcal{R}_{\left.u^{\infty}(T)\right)}$.

Proof. Let $k$ be as in Definition 2.2 (iii). Define $g_{k} \pi=k^{\beta}$, where $\pi: T^{\infty} \rightarrow$ $T^{\infty} / \mathcal{R}_{U^{\infty}(T)}$ is the quotient map. Then $g_{k}$ is a continuous function on $T^{\infty} / \mathcal{R}_{\mathcal{U}^{\infty}(T)}$, since $k \in \mathcal{U}^{\infty}(T)$ (Example 3.2 (iii)). Suppose now that, $\left(y_{n}\right)_{n=1}^{\infty}, v \in T^{\infty}$ be as in Remark 4.19. Then $\pi(v) \notin g_{l}^{-1}(\infty)$, sincel $l^{\beta}(v)=\infty$ and $g_{l} \pi=l^{\beta}$. On the other hand, $k^{\beta}(v)=1$, since $z\left(y_{n}\right)=0$ (see Definition 2.2 (iii)). But $\left(g_{l}^{-1}(\infty)\right)^{2} \subseteq$ $\left(T^{\infty} / \mathcal{R}_{U^{\infty}(T)}\right)^{2}$, an application of Corollary 2.6 then shows that $g_{k}\left(g_{l}^{-1}(\infty)\right)^{2}=$ 
0. Hence, $\pi(v) \notin c l\left(g_{l}^{-1}(\infty)\right)^{2}$, which implies the desired conclusion.

Recall that by Definition $2.2(i), c^{\beta}$ is a continuous homomorphism on $T^{\infty}$, and $h=1 / c, h \in \mathcal{U}^{\infty}(T)$ (Example $3.2(\mathrm{i})$ ). Let $\pi: T^{\infty} \rightarrow T^{\infty} / \mathcal{R}_{\mathcal{U}^{\infty}(T)}$ be the quotient map, and let $g_{c} \pi=c^{\beta}$. Then $g_{c}$ is a continuous homomorphism on $T^{\infty} / \mathcal{R}_{U^{\infty}(T)}$. Let $\mu^{(k)}, v$ be as in Remark 4.19. Then $c^{\beta}\left(\mu^{(k)}\right)=k, c^{\beta}(v)=\infty$, and so we obtain that $g_{c}$ maps $T^{\infty} / \mathcal{R}_{U^{\infty}(T)}$ onto the one-point compactification $\mathbb{N} \cup\{\infty\}$.

The proof of the following proposition is essentially the same as that of Proposition4.20.

Proposition 4.21. $\left(g_{c}^{-1}(\infty)\right)^{2}$ is not dense in $g_{c}^{-1}(\infty)$ (and hence is not dense in $T^{\infty} / \mathcal{R}_{\left.\mathcal{U}^{\infty}(T)\right) \text {. }}$

Theorem 4.22. Let $\pi: T^{\infty} \rightarrow T^{\infty} / \mathcal{R}_{U^{\infty}(T)}$ be the quotient map. Then the set $\left\{\pi(\xi): \xi \in T^{\infty}, l^{\beta}(\xi)<\infty\right\}$ is not dense in $T^{\infty} / \mathcal{R}_{\mathcal{U}^{\infty}}(T)$.

Proof. Let $\left(y_{n}\right)_{n=1}^{\infty}, v \in T^{\infty}$ be as in Remark 4.19. Put $Y=\left\{y_{n}: n \in \mathbb{N}\right\}$ and let $1_{Y}$

be the indicator function of $Y$. Then $1_{Y}^{\beta}(v)=1$. We complete the proof by showing that $1_{Y} \in \mathcal{U}^{\infty}(T)$. To see this, suppose that $\mu \in T^{\infty}, \eta \in\left(T \cup T^{\infty} \cup T T^{\infty}\right)$ and let $x_{\alpha} \rightarrow \mu$ for some net $\left(x_{\alpha}\right)$ in $T$ with supp $x_{\alpha} \rightarrow \infty$. If $\eta=t \in T$, then eventually supp $t<\operatorname{supp} x_{\alpha}$ and for such $\alpha$, eventually supp $x_{\alpha}<\operatorname{supp} y_{n}$, so that eventually $t x_{\alpha} \notin Y$. Hence $1_{Y}^{\beta}(t \mu)=0$.If $\eta \in T^{\infty}$ and $y_{\beta} \rightarrow \eta$ for some $\operatorname{net}\left(y_{\beta}\right)$ inTwith $\operatorname{suppy}_{\beta} \rightarrow \infty$, then $1_{Y}^{\beta}(\mu v)=\lim _{\alpha} \lim _{\beta} 1_{Y}\left(x_{\alpha} y_{\beta}\right)=0$ by a similar reason. Finally, if $\eta=t \lambda, \lambda \in T^{\infty}$. Then $\mu \lambda \in T^{\infty}$, since $T^{\infty}$ is a semigroup and hence $1_{Y}^{\beta}(\mu \eta)=1_{Y}^{\beta}(t \mu \lambda)=0$. Consequently, $1_{Y} \in \mathcal{U}^{\infty}(T)$. Let $g_{1_{Y}} \pi=1_{Y}^{\beta}$. Then $g_{1_{Y}}$ is continuous on $T^{\infty} / \mathcal{R}_{U^{\infty}(T)}$. Take $\xi \in T^{\infty}$ with $l^{\beta}(\xi)=k, k \in \mathbb{N}$. There exists a net $\left(z_{\gamma}\right)$ in $T$ such that $z_{\gamma} \rightarrow \xi$ with $\operatorname{supp} z_{\gamma} \rightarrow \infty$. It follows that,eventually $l\left(z_{\gamma}\right)=k$, hence eventually $z_{\gamma} \notin Y$. Therefore $1_{Y}^{\beta}(\xi)=0$, so $g_{1_{Y}} \pi(\xi)=0$. On the other hand, $g_{1_{Y}} \pi(v)=1_{Y}^{\beta}(v)=1$ and hence $\pi(v) \notin \operatorname{cl}\left\{\pi(\xi): \xi \in T^{\infty}, l^{\beta}(\xi)<\infty\right\}$, which implies the desired conclusion. 
For remainder of this section we consider the more general results of deseribing about inclusion between $\mathcal{U}^{\infty}(T)$ and $W A P^{\infty}(T)$ (see[1], for more details).

Theorem 4.23. For $f \in C(T)$, if $(\mu, v) \rightarrow f^{\beta}(\mu v): T^{\infty} \times\left(T \cup T^{\infty} \cup T T^{\infty}\right) \rightarrow \mathbb{C}$ is separately continuous, then $(\mu, v, \eta) \rightarrow f^{\beta}(\mu \nu \eta): T^{\infty} \times\left(T \cup T^{\infty} \cup T T^{\infty}\right) \times$ $T^{\infty} \rightarrow \mathbb{C}$ is also separately continuous.

Proof. Let $\left(\mu_{\alpha}\right)$ is a net in $T^{\infty}$ converging to $\mu \in T^{\infty}$ and let $v \in\left(T \cup T^{\infty} \cup T T^{\infty}\right)$, $\eta \in T^{\infty}$.

(i) If $v \in T^{\infty}$, then as is readily verified that $f^{\beta}\left(\mu_{\alpha} v \eta\right) \rightarrow f^{\beta}(\mu \nu \eta)$, since $v \eta \in T^{\infty}$ and $(\mu, v) \rightarrow \mu v: \beta T \times T^{\infty} \rightarrow \beta T$ is right continuous.

(ii) If $v=t \lambda, t \in T$ and $\lambda \in T^{\infty}$. Then $\lambda \eta \in T^{\infty}$ since $T^{\infty}$ is semigroup and $t \mu_{\alpha} \rightarrow t \mu$ inT.Using Definition 3.5[1] and that $(\mu, v) \rightarrow \mu v: \beta T \times T^{\infty} \rightarrow \beta T$ is right continuous, it follows that $f^{\beta}\left(\mu_{\alpha} v \eta\right) \rightarrow f^{\beta}(\mu v \eta)$.

(iii) If $v=t \in T$, then by a similar argument, $f^{\beta}\left(\mu_{\alpha} v \eta\right) \rightarrow f^{\beta}(\mu \nu \eta)$. Let $\left(\eta_{\alpha}\right)$ isanetin $T^{\infty}$ converging to $\eta \in T^{\infty}$. Take $\mu \in T^{\infty}, v \in\left(T \cup T^{\infty} \cup T T^{\infty}\right)$. (i) Let $v \in T^{\infty}$. Then $\mu \nu \in T^{\infty}$.In fact $\eta_{\alpha} \rightarrow \eta$ in $\left(T \cup T^{\infty} \cup T T^{\infty}\right)$ and by hypothesis, $(\mu, v) \rightarrow f^{\beta}(\mu \nu)$ is separately continuous on $T^{\infty} \times\left(T \cup T^{\infty} \cup\right.$ $\left.T T^{\infty}\right)$, from which it follows that $f^{\beta}\left(\mu \nu \eta_{\alpha}\right) \rightarrow f^{\beta}(\mu \nu \eta)$.

(ii) Let $v=t \lambda, t \in T$ and $\lambda \in T^{\infty}$. Then $\mu \lambda \in T^{\infty}$. Indeed, $t \eta_{\alpha} \rightarrow t \eta$ in $\left(T \cup T^{\infty} \cup\right.$ $\left.T T^{\infty}\right) \subseteq \beta T$ and $(\mu, v) \rightarrow f^{\beta}(\mu \nu)$ is separately continuous on $T^{\infty} \times\left(T \cup T^{\infty} \cup\right.$ $\left.T T^{\infty}\right)$, hence $f^{\beta}\left(\mu \nu \eta_{\alpha}\right) \rightarrow f^{\beta}(\mu \nu \eta)$.

(iii) Let $v=t \in T$. The proof is similar.

Finally, suppose that $\left(v_{\alpha}\right)$ is a net $\operatorname{in}\left(T \cup T^{\infty} \cup T T^{\infty}\right)$ converging to $v \in\left(T \cup T^{\infty} \cup T T^{\infty}\right)$, and let $\mu, \eta \in T^{\infty}$. Then $v_{\alpha} \eta \rightarrow v \eta \operatorname{in}\left(T \cup T^{\infty} \cup T T^{\infty}\right)$. But $(\mu, v) \rightarrow f^{\beta}(\mu \nu)$ is separately continuous on $T^{\infty} \times\left(T \cup T^{\infty} \cup T T^{\infty}\right)$, hence $f^{\beta}\left(\mu v_{\alpha} \eta\right) \rightarrow f^{\beta}(\mu \nu \eta)$. This proves our assertion.

Theorem4.24. For $f \in C(T)$, let $\quad \operatorname{both}(\mu, v) \rightarrow f^{\beta}(\mu v): T^{\infty} \times\left(T \cup T^{\infty}\right) \rightarrow \mathbb{C}$ and $(\mu, v, \eta) \rightarrow f^{\beta}(\mu \nu \eta): T^{\infty} \times\left(T \cup T^{\infty}\right) \times T^{\infty} \rightarrow \mathbb{C}$ are separately continuous. Then $f \in W_{2}^{\infty}(T)$.

Proof. We use Theorem 3.11[1]. Suppose $\mu, v, \eta \in T^{\infty}$, and $\left(x_{\alpha}\right)$ isanet inTconverging to $\mu$ with $\operatorname{supp} x_{\alpha} \rightarrow \infty$. Then $f^{\beta}\left(x_{\alpha} \eta v\right) \rightarrow f^{\beta}(\mu \eta v)$,since $(\mu, v) \rightarrow \mu v: \beta T \times T^{\infty} \rightarrow$ $\beta T$ is right continuous, and $\eta v \in T^{\infty}$. By hypothesis, $(\mu, v) \rightarrow f^{\beta}(\mu v)$ is separately continuous on $T^{\infty} \times\left(T \cup T^{\infty}\right)$, hence from Remark 4.2, and that $T^{\infty}$ is a semigroup we see that 


$$
f^{\beta}(\mu \eta v)=f^{\beta}(v \mu \eta)=f^{\beta}(\eta v \mu), f^{\beta}(\eta \mu \nu)=f^{\beta}(\nu \eta \mu)=f^{\beta}(\mu v \eta) .
$$

On the other hand, $\lim _{\alpha} f^{\beta}\left(x_{\alpha} \eta v\right)=\lim _{\alpha} f^{\beta}\left(\eta x_{\alpha} v\right)=f^{\beta}(\eta \mu v)$, since $(\mu, v, \eta) \rightarrow$ $f^{\beta}(\mu v \eta)$ is separately continuous on $T^{\infty} \times\left(T \cup T^{\infty}\right) \times T^{\infty}$ and $x_{\alpha} \rightarrow \mu^{\text {in }}$ $\left(T \cup T^{\infty}\right)$. Thus $f^{\beta}(\mu \eta v)=f^{\beta}(\eta \mu \nu)$, and we have that $f \in W_{2}^{\infty}(T)$, as desired.

Definition 3.1and the preceding theorems now imply that the following result.

Corollary 4.25. Let $T$ be a commutative standard oid. Then $\mathcal{U}^{\infty}(T) \subseteq$ $W_{2}^{\infty}(T)$.

Corollary 4.26. Let The a commutative standard oid. Then $\mathcal{U}^{\infty}(T) \subseteq$ $W A P^{\infty}(T)$.

The proof now follow by the preceding Corollary,Remark4.2 and Definition 4.1[1].

Example 4.27. We have already seen that $\mathcal{U}^{\infty}(T) \subseteq W A P^{\infty}(T)$. Now we show that the converse is not true. For this purpose, consider two sets $E=\left\{(x(n))_{n \in \mathbb{N}} \in T: x(1)=1\right\}$ and $F=\left\{(x(n))_{n \in \mathbb{N}} \in T: x(1)=\infty\right\}$. Then $T=E \cup F$. Now let $\left(u_{2 m}\right)_{m \in \mathbb{N}}$ and $\left(u_{2 n+1}\right)_{n \in \mathbb{N}}$ be two sequences in $T$ such that $u_{2 m}, u_{2 n+1} \in U$ for all $m, n$. Takeu $u_{1} \in U$. PutD $=\left\{u_{1} u_{2 m} u_{2 n+1}: m>n\right\} \subseteq F$. Define $f: T \rightarrow \mathbb{C} b y$

$$
f(x)=\left\{\begin{array}{ll}
1 / c(x) & , \quad x \in E \\
1 & , \quad x \in D \\
0 & , \quad \text { otherwise }
\end{array} \quad(x \in T)\right.
$$

Then $f \in W_{1}^{\infty}(T)$ (Example 3.7[1]). We show that $f \in W_{2}^{\infty}(T)$ (Definition 3.9[1]). Assume that $\left(x_{\alpha}\right),\left(y_{\beta}\right)$ and $\left(z_{\gamma}\right)$ be nets in $T$ withsupp $x_{\alpha} \rightarrow \infty$, supp $y_{\beta} \rightarrow \infty$ and supp $z_{\gamma} \rightarrow \infty$. Then (for all sufficiently large $\left.\alpha, \beta, \gamma\right) x_{\alpha}(1)=$ $1, y_{\beta}(1) a n d z_{\gamma}(1)=1$, so that $x_{\alpha}, y_{\beta}, z_{\gamma} \in E$. It is therefore easy To verify that $f \in W_{2}^{\infty}(T)$, since $h=1 / c \in W_{2}^{\infty}(T)$ (Example 3.10[1])hence $f \in W A P^{\infty}(T)$. To prove that $f \notin \mathcal{U}^{\infty}(T)$, we remind the reader that for any $f \in \mathcal{U}^{\infty}(T), f^{\beta}(t \mu v)=f^{\beta}(t v \mu)$ wheret $\in$ Tand $\mu, v \in T^{\infty}$. We assume that $\mu, v$ be the cluster points of the sequences $\left(u_{2 m}\right)_{m \in \mathbb{N}},\left(u_{2 n+1}\right)_{n \in \mathbb{N}}$ in $\beta T$ 
respectively. Then $\mu, v \in T^{\infty}$, since supp $u_{2 m} \rightarrow \infty$ and supp $u_{2 n+1} \rightarrow \infty$.

Hence, $\lim _{m} \lim _{n} f\left(u_{1} u_{2 m} u_{2 n_{+}+}\right)=0, \lim _{n} \lim _{m} f\left(u_{1} u_{2 m} u_{2 n_{+} 1}\right)=1$, so that Both iterated limits of $f\left(u_{1} u_{2 m} u_{2 n_{+}+}\right)$exist. But $\lim _{m} \lim _{n} f\left(u_{1} u_{2 m} u_{2 n_{+} 1}\right)=$ $f^{\beta}\left(u_{1} \mu v\right)$, and $\lim _{n} \lim _{m} f\left(u_{1} u_{2 m} u_{2 n+1}\right)=f^{\beta}\left(u_{1} v \mu\right)$, which is clearly impossible. Thus $f \notin \mathcal{U}^{\infty}(T)$, asclaimed.

\section{Idempotents}

Recall that $T^{\infty}$ is a compact right topological semigroup and $\mathcal{R}_{\mathcal{U}^{\infty}(T)}$ is a relation on $T^{\infty}$ (Definition 4.1). Then $\mathcal{R}_{\mathcal{U}^{\infty}(T)}$ is congruence and under certain topology on $T^{\infty}$, is also a closed relation, so that the quotient space $T^{\infty} / \mathcal{R}_{U^{\infty}}(T)$ is a compact topological semigroup (Corollary 4.5), which is commutative (Corollary 4.6). In this section we are concerned with obtaining of idempotents $T^{\infty} / \mathcal{R}_{U^{\infty}(T)}$. In connection with the present section special suboids of an oid Tplay an important role. Each special suboid corresponds to a strictly increasing sub sequence of $\mathbb{N}$.A more details analysis of special suboids can be found in[1]. For an infinite subset $A \subseteq \mathbb{N}$, the special suboid of an oid $T$ corresponding to The strictly increasing sequence of $A$ is denoted by $S(A)$. Then $S(A)$ produces a compact right topological semigroup $S^{\infty}(A)$ which is a sub semigroup of $T^{\infty}$. Indeed, $\pi\left(S^{\infty}(A)\right)$ is a compact commutative sub semigroup of ${ }^{\infty}($ $T^{\infty} / \mathcal{R}_{\mathcal{U}^{\infty}(T)}$, where $\pi: T^{\infty} \rightarrow T^{\infty} / \mathcal{R}_{U^{\infty}(T)}$ is the quotient map.

Proposition5.1. Let $A \subseteq \mathbb{N}$ be an in finite set and let $1_{S(A)}$ be the indicator function of $S(A)$. Then $1_{S(A)}^{\beta}(\mu v)=1_{S(A)}^{\beta}(\mu) 1_{S(A)}^{\beta}(v)$ for all $\mu \in T^{\infty}$ and $v \in(T \cup$ $\left.T^{\infty} \cup T T^{\infty}\right)$.

Proof. It is a straightforward argument that $1_{S(A)}(x y)=1_{S(A)}(x) 1_{S(A)}(y)$ For $x, y \in T$ such that $(\operatorname{supp} x) \cap(\operatorname{supp} y)=\emptyset$. Suppose that $t \in T, \mu \in T^{\infty}$ such that $x_{\alpha} \rightarrow \mu$ for some net $\left(x_{\alpha}\right)$ in $T$ with $\operatorname{supp} x_{\alpha} \rightarrow \infty$. Then even-

Tually supp $t<\operatorname{supp} x_{\alpha}$, so that eventually $1_{S(A)}\left(t x_{\alpha}\right)=1_{S(A)}(t) 1_{S(A)}\left(x_{\alpha}\right)$.

Since $\{0,1\}$ is a compact commutative semigroup with the usual multiplication, and $t \mu=\lim _{\alpha} t x_{\alpha}$, it follows that $1_{S(A)}^{\beta}(t \mu)=1_{S(A)}(t) 1_{S(A)}^{\beta}(\mu)$. Now let $v \in T^{\infty}$ with $y_{\beta} \rightarrow v$ for some net $\left(y_{\beta}\right)$ inTsuch that supp $y_{\beta} \rightarrow \infty$.

Then by a similar reason, we see that

$$
1_{S(A)}^{\beta}(\mu v)=\lim _{\alpha} \lim _{\beta} 1_{S(A)}\left(x_{\alpha} y_{\beta}\right)=\lim _{\alpha} 1_{S(A)}\left(x_{\alpha}\right) 1_{S(A)}^{\beta}(v)=1_{S(A)}^{\beta}(\mu) 1_{S(A)}^{\beta}(v) .
$$


Finally if $v=t \lambda, t \in T, \lambda \in T^{\infty}$, then $\mu \lambda \in T^{\infty}$, and $\mu \nu=\mu t \lambda=t \mu \lambda$. Thus, by above paragraph, we obtain that $1_{S(A)}^{\beta}(\mu \nu)=1_{S(A)}^{\beta}(\mu) 1_{S(A)}^{\beta}(v)$, as desired.

Corollary5.2. For an infinite set $A \subseteq \mathbb{N}$, the indicator function $1_{S(A)}$ of $S(A)$ is in $\mathcal{U}^{\infty}(T)$.

Proof. To show that $1_{S(A)} \in \mathcal{U}^{\infty}(T)$, it is adequate by Theorem 3.7, to show that $v \rightarrow L_{v} f^{\beta}:\left(T \cup T^{\infty} \cup T T^{\infty}\right) \rightarrow C\left(T^{\infty}\right)$ is norm-continuous. Assume that $\left(v_{\alpha}\right)$ be any net in $\left(T \cup T^{\infty} \cup T T^{\infty}\right)$ such that $v_{\alpha} \rightarrow v$ in $\left(T \cup T^{\infty} \cup T T^{\infty}\right)$. Then $1_{S(A)}^{\beta}\left(v_{\alpha}\right) \rightarrow 1_{S(A)}^{\beta}(v)$. Thus for $\varepsilon>0$, there exists $\alpha_{0}$ such that for $\alpha \geq \alpha_{0},\left|1_{S(A)}^{\beta}\left(v_{\alpha}\right)-1_{S(A)}^{\beta}(v)\right|<\varepsilon / 2$. However, by definition of $1_{S(A)}$, we have $1_{S(A)}^{\beta}(\mu) \in\{0,1\}$ for all $\mu \in T^{\infty}$, which is clearly that from Proposition 5.1, and for $\alpha \geq \alpha_{0}$,

$\left|1_{S(A)}^{\beta}(\mu) 1_{S(A)}^{\beta}\left(v_{\alpha}\right)-1_{S(A)}^{\beta}(\mu) 1_{S(A)}^{\beta}(v)\right|=\left|1_{S(A)}^{\beta}(\mu)\right|\left|1_{S(A)}^{\beta}\left(v_{\alpha}\right)-1_{S(A)}^{\beta}(v)\right|<$ $\varepsilon / 2\left(\mu \in T^{\infty}\right)$. Hence, for $\alpha \geq \alpha_{0},\left\|L_{\mu_{\alpha}} 1_{S(A)}^{\beta}-L_{\mu} 1_{S(A)}^{\beta}\right\|<\varepsilon$ and the proof of the corollary is complete.

Proposition5.3.If $A, B$ be two infinite subsets of $\mathbb{N}$ with $A \cap B$ is finite. Then $\pi\left(S^{\infty}(A)\right) \cap \pi\left(S^{\infty}(B)\right)=\emptyset$, where $\pi: T^{\infty} \rightarrow T^{\infty} / \mathcal{R}_{U^{\infty}(T)}$ is quotient map. Proof. This uses Corollary 5.2, the proof is essentially the same as that of Proposition7.1[1].

We finish the present section by giving the following main result. Inconnection with this result we will use non-principal ultrafilters on $\mathbb{N}$. We remind the reader that, if $\mathcal{F}$ is a non-principal ultrafilter on $\mathbb{N}$ and $A \in \mathcal{F}$, Then $A$ is an infinite set. Moreover, the number of non-principal ultrafilters on $\mathbb{N}$ is $2^{c}$ [8].

Here is the main result of this section.

Theorem5.4. $T^{\infty} / \mathcal{R}_{\mathcal{U}^{\infty}(T)}$ contains at least $2^{c}$ idempotents.

Proof. This uses Proposition5.3, the proof is parallel to that of Theorem $7.2[1]$.

\section{Free abelian groups}

In connection with Theorem 4.7, of the Section 4, it should be mentioned that, it is possible for a compact commutative topological semigroup $T^{\infty} / \mathcal{R}_{\mathcal{U}^{\infty}(T)}$ have a minimal idempotent with a unique minimal left ideal and a unique minimal right ideal,so that $K\left(T^{\infty} / \mathcal{R}_{U^{\infty}(T)}\right)$, the minimal ideal of $T^{\infty} / \mathcal{R}_{U^{\infty}(T)}$ is a maximal group(see[3],for more details). The aim of this Section is the search for existence in $K\left(T^{\infty} / \mathcal{R}_{U^{\infty}(T)}\right)$ a free abelian group on $2^{c}$ generator. Let us first give the following result. 
Lemma6.1.Let $\psi$ is an arbitrary function from $\mathbb{N}$ to $\mathbb{R}$, let $f: T \rightarrow \mathbb{C}$ be defined by $f(t)=\exp i \sum\{\psi(n): n \in \operatorname{supp} t\}$. Then $f \in \mathcal{U}^{\infty}(T)$.

Proof. It is easily seen that $f^{\beta}(t \mu)=f(t) f^{\beta}(\mu)$ for $t \in T, \mu \in T^{\infty}$, since $f$ is an oid-map (that is, $f(s t)=f(s) f(t) \quad$ whenever $s, t \in T$ with (supps) $\cap$ $(\operatorname{supp} t)=\emptyset)$.Moreover, $f^{\beta}$ is a homomorphism of $T^{\infty}$ to the circle group $\mathbb{T}$. Thus $f^{\beta}(\mu v)=f^{\beta}(\mu) f^{\beta}(v)$ for $\mu \in T^{\infty}, v \in\left(T \cup T^{\infty} \cup T T^{\infty}\right)$. To prove that $f \in \mathcal{U}^{\infty}(T)$, we show that $v \rightarrow L_{v} f^{\beta}:\left(T \cup T^{\infty} \cup T T^{\infty}\right) \rightarrow C\left(T^{\infty}\right)$ is normcontinuous(Theorem 3.7). Now suppose that $\left(v_{\alpha}\right)$ be any net $\operatorname{in}\left(T \cup T^{\infty} \cup\right.$ $\left.T T^{\infty}\right)$ with $v_{\alpha} \rightarrow v$ in $\left(T \cup T^{\infty} \cup T T^{\infty}\right)$.

Then $f^{\beta}\left(v_{\alpha}\right) \rightarrow f^{\beta}(v)$, hence for $\varepsilon>0$, there exists $\alpha_{0}$ such that for $\alpha \geq \alpha_{0},\left|f^{\beta}\left(v_{\alpha}\right)-f^{\beta}(v)\right|<\varepsilon / 2$. But since, $\left|f^{\beta}(\mu)\right|=1$ for all $\mu \in \beta T$, it follows directly that for $\alpha \geq \alpha_{0}$ and for all $\mu \in T^{\infty}$,

$\left|f^{\beta}(\mu) f^{\beta}\left(v_{\alpha}\right)-f^{\beta}(\mu) f^{\beta}(v)\right|=\left|f^{\beta}(\mu)\right|\left|f^{\beta}\left(v_{\alpha}\right)-f^{\beta}(v)\right|<\varepsilon / 2$. Thus, for $\alpha \geq \alpha_{0},\left\|L_{v_{\alpha}} f^{\beta}-L_{v} f^{\beta}\right\|<\varepsilon$, that is $f \in \mathcal{U}^{\infty}(T)$.

We remind the reader that, if $M=\left\{\mu \in T^{\infty}: c^{\beta}(\mu)=1\right\}$, then it is easy to verify that $M=\left\{\mu \in T^{\infty}: \mu \in \operatorname{cl}\left\{u_{m}: m \in \mathbb{N}\right\}\right\}$, and $\operatorname{card}(M)=2^{c}$ (see [2],

Remark5.8). Recall that, $\pi: T^{\infty} \rightarrow T^{\infty} / \mathcal{R}_{\mathcal{U}^{\infty}(T)}$ is the quotient map which is a Continuous epimorphism. We now give the following result which is the last major result of this section.

Theorem6.2. $K\left(T^{\infty} / \mathcal{R}_{\mathcal{U}^{\infty}(T)}\right)$ contains a free abelian group on $2^{c}$ generators. Proof. This use Lemma6.1 and Corollary 4.26, the proof is similar to that of Theorem 8.1[1].

\section{References}

[1] A.M. Aminpour, "Spaces of functions determind by iterated limits "at infinity"on an oid", Proc. Cambridge Phill. Soc.111(1992), 127-142.

[2] A. M. Aminpour, "A sub semigroupof some Stone-Čech compactification”, Math. Nachr. 158(1992), 207-218.

[3] J.F. Berglund,H.D.Junghennand P.Milnes, “Analysis on semigroups”, Wiley, New York(1989).

[4] P. Civin and B. Yood,"The second conjugate space of a Banach algebra as an algebra", Pacific J.Math.11(1961), 847-870. 
A.M. Aminpour, M. Seilani / J. Math. Computer Sci. 12 (2014), 219-234

[5] N. Hindman and J.S. Pym, "Free groups and semigroups in $\beta \mathbb{N}$ ". Semigroup Forum 30(1984), 177-193.

[6] T. Papazyan, "Oids, finite sums and the structure of the Stone-Čech compactification of a discrete semigroups",SemigroupForum42(3)(1991), 265-277.

[7] J.S. Pym, "Semigroup structure in Stone-Čech compactification”.J.London. Math. Soc.(2)36(1987), 421-428.

[8] R.C. Walker,"The Stone-Čech compactification”, Springer-Verlag,Berlin (1974).

[9]J.L.Kelley, “General topolog”, Van Nostrand(1955).

[10 ]J.F. Berglund,H.D. Junghenn and P. Milnes, “Compact right topological semigroups and Generalization of almost periodicity ",LectureNotes in Math.Vol.663,Springer-Verlag, Berlin, (1973). 
